\title{
The Construction of a Graphene Hall Effect Magnetometer
}

\author{
Davut Izci, Carl Dale, Neil Keegan, John Hedley
}

\begin{abstract}
This paper deals with design and fabrication of graphene micro-Hall devices along with driving and processing electronics to construct a highly sensitive graphene magnetometer system. Graphene micro-Hall elements were fabricated by implementing microfabrication techniques. Custom driving and processing circuitry was designed and built on a printed circuit board (PCB) and integrated with fabricated graphene Hall devices; reducing the offset equivalent magnetic field and greatly improving the sensitivity. The performance parameters were quantitatively analyzed for characterizing the sensitivity in terms of linearity, current-related sensitivity, and magnetic field resolution. The potential of graphene magnetometers for detection capability of low fields has been demonstrated with a current related sensitivity of $2540 \mathrm{~V} / \mathrm{AT}$ and magnetic field resolution of $162 \mathrm{nT} / \sqrt{ } \mathrm{Hz}$.
\end{abstract}

Index Terms-Graphene, Hall effect, magnetic sensors, magnetometer, microfabrication, micro-Hall devices

\section{INTRODUCTION}

$\mathrm{M}$ AGNETIC SENSORS have been useful tools for human beings to drive, store, analyze and utilize in control systems. For example, magnetic storage disks in computers, non-contact reliable switches in airplanes and automobiles, and control systems in factories for reliable and higher productivity [1]. There are several forms of magnetic sensors in terms of their principle of operations and most commonly used classes can be listed as magnetoresistive [2], superconducting quantum interference device (SQUID) [3], spin-valve [4], and Hall effect sensors [5]. There are examples with excellent sensitivity [6], however implementation can be difficult due to specific requirements, such as fabrication difficulties, operating conditions and specific temperature requirements [1].

Hall devices are commonly used magnetic sensors and they operate on the principle of the Lorentz force [5]. The

Manuscript submitted for review on April 6, 2018, reviewed on Jun 20, 2018 and revised on August 2, 2018. This is an expanded paper from the IEEE SENSORS 2017 Conference. Data supporting this publication is openly available under an 'Open Data Commons Open Database License'. Additional metadata are available at: http://dx.doi.org/10.17634/101566-2. The authors acknowledge the EPSRC for financial support for the work (EP/I015930/1, $\mathrm{EP} / \mathrm{K} 031953 / 1$.

Davut Izci and John Hedley are with the School of Engineering, Microsystems Research Group, Newcastle University, Newcastle upon Tyne, NE1 7RU, United Kingdom (e-mail: d.izci@newcastle.ac.uk davutizci@gmail.com; john.hedley@newcastle.ac.uk).

Carl Dale and Neil Keegan are with the Institute of Cellular Medicine, Diagnostics and Therapeutic Technologies, Newcastle University, Newcastle upon Tyne, NE2 4HH, United Kingdom (e-mail: carl.dale@newcastle.ac.uk; neil.keegan@newcastle.ac.uk). advantage of developing Hall effect magnetic sensors is that they are relatively easy to fabricate when compared to the other magnetic device classes. More importantly, the ultimate goal of developing a sensor that can be commercialized with a translational application is achievable due to the ease of electronic integration.

Devices based on the Hall effect have been investigated for more than a hundred years [5] with deployment in cars, planes, machine tools, computers and medical equipment [7]. The devices have led to a variety of applications [8]; from antilock braking systems in vehicles, disk drives in computers to highly reliable position sensing with automated systems in factories. In the last decade, there has been a considerable number of reports utilizing the Hall principle for biosensing applications [9-15]. In terms of industrial importance, Hall devices are the most widely used magnetic sensor format [16] due to their ease of fabrication and implementation, small size and high linearity [17].

Materials with high carrier mobility, low carrier concentration and narrow band gaps are desirable for Hall effect applications since these properties provide the exact characteristics for high sensitivity Hall devices [5]. In addition, reducing the material thickness provides an exceptional advantage since the charge carriers are confined, which produces a stronger force [8].

Recently, graphene has become a material of interest for many applications. It is a one-atom thick nanomaterial consisting of carbon atoms formed in a hexagonal honeycomb shape [18]. There have been many reported studies for a wide range of applications $[18,19]$. The unique material properties of graphene make it a promising candidate for mechanical, electrical and optical systems [20-23]. Possessing ultra-high carrier mobility [24] and being one-atom thick makes graphene a specifically unique material for Hall effect type applications $[25,26]$ since charge carriers are constrained in a two-dimensional plane thus providing a higher sensitivity and an outstanding resolution. In addition, graphene is also an intrinsically low noise material [27]. Therefore, there has been a number of publications exploring the potential of graphene as a Hall sensor [28-32] where devices having superior sensitivities with lower noise were obtained. The sensitivity, linearity and temperature stability of graphene Hall effect sensors have been demonstrated to be incredibly better than commercially available Hall sensors [7, 27].

In general, it is relatively simple to integrate Hall devices with on-chip circuitry for actuation and read-out, however, this is not straightforward when using graphene materials. The theoretical sensitivity of the system reduces significantly during implementation - as demonstrated by a previous 
attempt to integrate Hall effect graphene devices on a CMOS chip [33]. In the reported work, the achieved sensitivity limit $(200 V / A T)$ is similar to silicon based Hall devices $(310 \mathrm{~V} / A T)$ [34]. This may be due to introducing defects in the graphene during the processing steps. Therefore, our research team revisited the concept in this work. The aim was to design and fabricate micro Hall effect graphene devices for use as high sensitivity magnetometers, whilst integrating it with the required biasing and read-out circuitry.

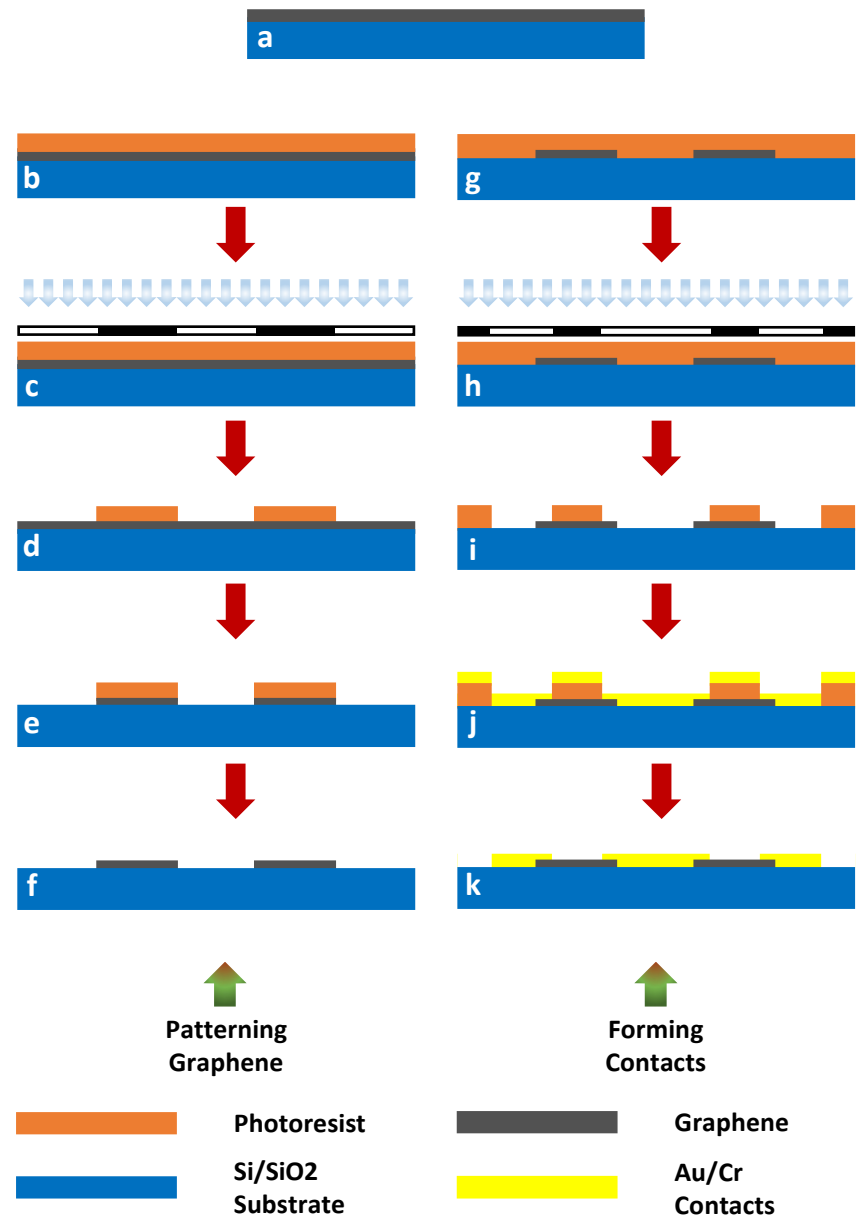

Fig. 1. Schematic for microfabrication process to form graphene micro-Hall devices. (a) High quality CVD grown graphene situated on a $\mathrm{Si} / \mathrm{SiO} 2$ wafer. (b) The wafer was covered with AZ5214E photoresist and pre-baked at $90^{\circ} \mathrm{C}$ for 15 minutes. (c) UV exposure for 14 seconds using a patterned mask. (d) Developing the photoresist for obtaining relevant patterns. (e) Etching process via oxygen plasma to remove graphene not protected by the photoresist layer. (f) Photoresist removal. (g) Another layer of photoresist was spin-coated onto the sample and pre-baked. (h) Second UV exposure for defining contacts. (i) Photoresist development. (j) Chromium and gold evaporation. (k) Lift-off.

To address the issue, a bespoke circuitry was designed and constructed on a PCB board and integrated with the graphene Hall effect sensors for biasing and processing the output. The circuitry was used to correct the output by eliminating nondesired offset voltages and reducing noise levels thus providing an improvement on the sensitivity of the overall system. The ability of the devices and corresponding circuitry to detect low magnetic field ranges was assessed and performance of the devices compared with previously reported works.

\section{FABRICATION}

Microfabrication process started by directly processing CVD grown graphene without any protective layer. To help better adhesion of the graphene to the $\mathrm{Si} / \mathrm{SiO}_{2}$ substrate and prevent any delamination, the samples were annealed at a temperature of $300{ }^{\circ} \mathrm{C}$ under free flow of nitrogen for three hours. An array of single graphene Hall devices with an active area of $10 \mu \mathrm{m} \times 10 \mu \mathrm{m}$ were fabricated on $5 \mathrm{~mm} \times 5 \mathrm{~mm}$ dies using the following microfabrication process highlighted in Fig. 1 .

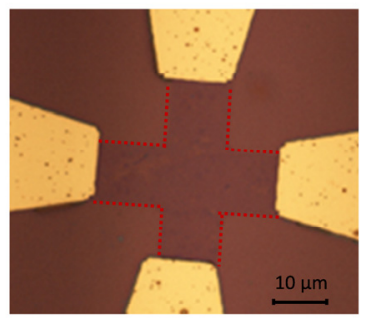

(a)

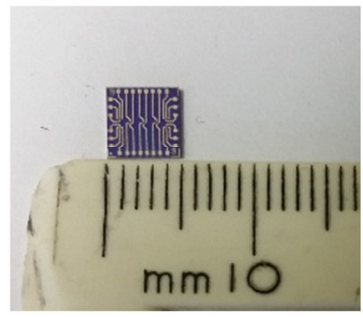

(b)
Fig. 2. An optical image of a graphene Hall effect device with $\mathrm{Cr} / \mathrm{Au}$ contacts. Graphene layer is highlighted with red-dotted lines (a). A view of one of the fabricated $5 \mathrm{~mm} \times 5 \mathrm{~mm}$ dies containing 7 devices (b).

A photoresist layer was spin coated to cover a 4-inch silicon wafer containing a $285 \mathrm{~nm}$ surface oxide layer with a CVD grown and transferred graphene layer (Graphene Supermarket). Desired patterns were obtained by implementation of a lithography process. The wafer then underwent a dry etching process via oxygen plasma for 13 minutes to remove the graphene not protected by photoresist. After removal of photoresist, a similar lithography process was performed for defining the contacts. A $30 \mathrm{~nm}$ layer of chromium and $250 \mathrm{~nm}$ layer of gold were deposited with electron beam evaporation following the lithography steps. The desired contact patterns were formed after the lift-off. Lastly, the processed wafer was diced into $5 \times 5 \mathrm{~mm}^{2}$ dies which include 7 individual micro-Hall elements.

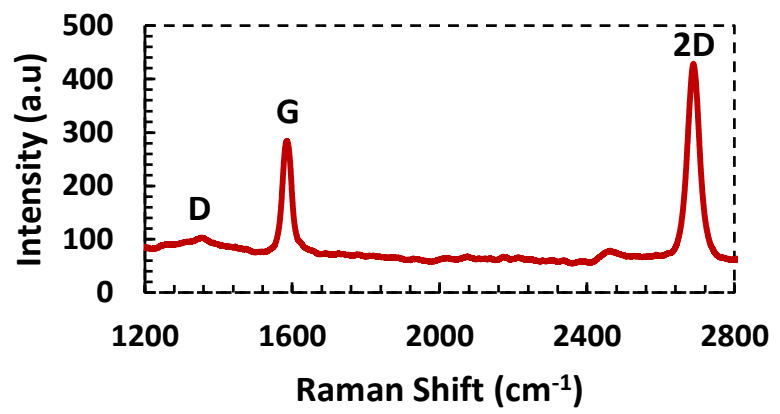

Fig. 3. The Raman spectrum of graphene after the microfabrication process.

Devices having an active area of $10 \mu \mathrm{m} \times 10 \mu \mathrm{m}$ were formed in a cross-like geometry as shown in Fig. 2. The length to width ratio was designed to be greater than 3 in order to avoid weakening the Hall effect due to geometrical factor [5]. To assess the quality of the graphene, Raman spectroscopy measurements were performed. The Raman data, shown in Fig. 3 , was obtained after completion of the fabrication procedure. The peaks characteristic to graphene were assessed. A sharp 2D peak and quite small $\mathrm{D}$ peak were obtained with a 
calculated $2 D / G$ intensity ratio of around 2.5 which indicates a high quality single layer graphene [35]. Although Raman spectrum confirms a generally high-quality film, it is evident from Fig. 2(a) that the fabrication process does introduce tears at certain locations in this graphene film.

\section{MEASUREMENT SETUP}

The simple rule for obtaining a Hall voltage is to form at least four contacts on a thin sheet of metal or semiconductor. This is, in principle, sufficient for capturing any Hall voltage which occurs due to the applied perpendicular magnetic field. However, Hall voltages are typically quite small and therefore require amplification. In addition, offset voltages may be significantly higher than the Hall voltage. The offset voltage is an undesired outcome that occurs due to contact misalignments or poor surface roughness and nonhomogeneous device structure [8]. This must be eliminated when forming a system with high sensitivity. Various options such as auto-zeroing, chopper stabilization and correlated double sampling are available [36-38] to be implemented for eliminating undesired effects. For this work, a 'current spin model' [39] was employed by designing and constructing a PCB circuit board to bias the devices and process the outputs. The schematic in Fig. 4 demonstrates the steps that were implemented for driving devices and reading the outputs.

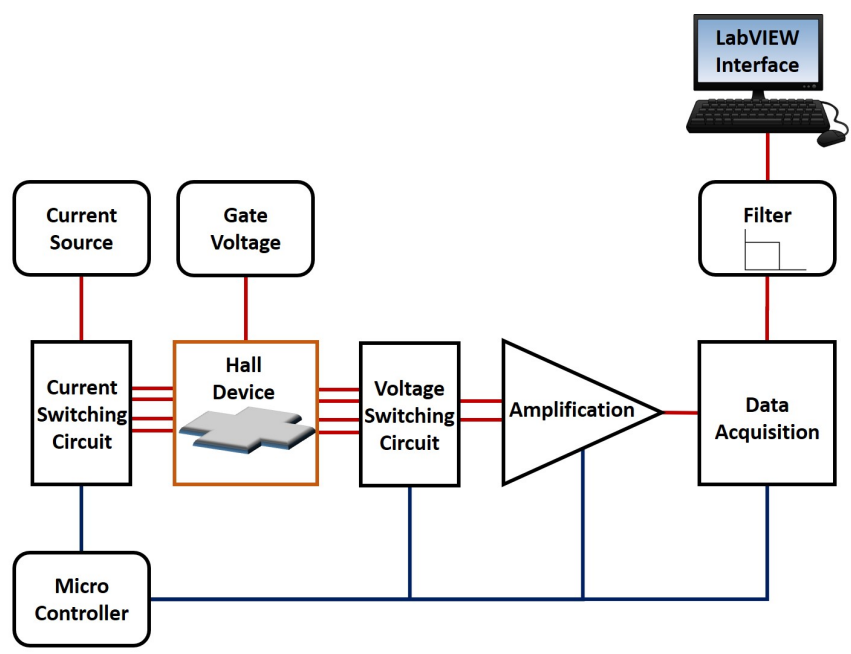

Fig. 4. Schematic of developed driving and processing circuitry for rotating the current between contacts and reading the output simultaneously. Current and voltage switching circuits are simultaneously operating with the help of a microcontroller and the output is amplified before being read by the data acquisition card. Then, the obtained output is filtered and visualized via a user interface created via LabVIEW. The entire system synchronously operates to provide a smooth elimination process.

Fundamentally, the current is driven from one of the contacts to a non-neighboring contact and simultaneously the produced output voltage is measured across the remaining two contacts (e.g. current flows from the top contact to the bottom and the voltage difference between left and right contacts is measured - see Fig. 2(a)). In such a case, the produced output includes both Hall voltage $\left(V_{H}\right)$ generated by applied perpendicular magnetic field $\left(B_{y}\right)$ and offset voltage $\left(V_{o f f}\right)$. To be able to remove the offset voltage, the current flow is rotated $90^{\circ}$ and passes through two new non-neighboring contacts whilst the voltage across the remaining contacts is measured. The current flows from left contact to the right contact and the voltage difference between bottom and top contacts are measured (see Fig. 2(a)). In this case, the measured output includes Hall voltage subtracted by offset voltage. In order for a more robust elimination, this procedure continues for one complete cycle meaning that the current is driven between all contacts for a complete cycle following $90^{\circ}$ rotation steps. Averaging the obtained outputs eliminates the offset and reveals the Hall voltage. A typical processed output of the device for one cycle is given in Fig. 5. Each region indicated in the figure corresponds to the specific cases mentioned above, e.g., region 1 is the measured output between left and right contacts whilst the current flows from top to bottom.

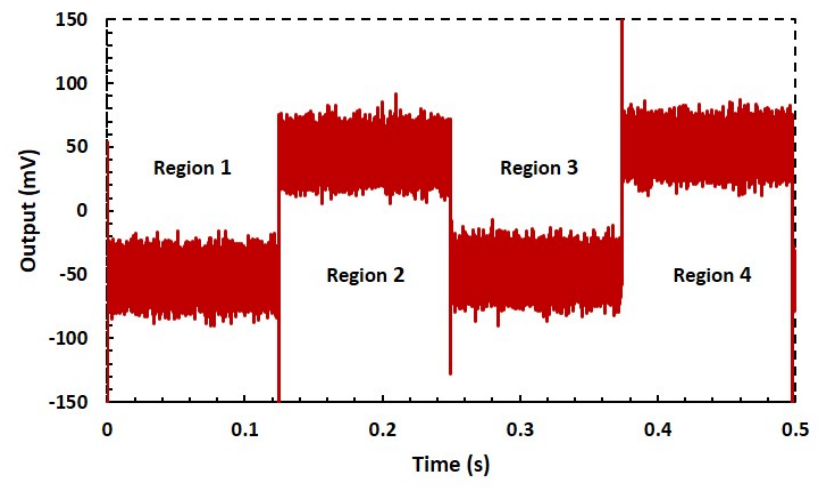

Fig. 5. A Typical output obtained after one cycle with a rotation frequency of $2 \mathrm{~Hz}$. Each region corresponds to a specific obtained output of which current flows between two non-neighboring contacts.

The image in Fig. 6 shows the designed system built on a PCB board. The constructed PCB has an on-board constant current source, which can easily be adjusted from $1 \mu \mathrm{A}$ to 10 $\mathrm{mA}$ and can bias the sensor in floating or grounded mode of operation. Due to the controllable sensitivity of graphene, an optional input for gate voltage was also included. Current and voltage switching mechanisms were connected to the current source and the amplifier along with the connections to device contacts. A microcontroller simultaneously manipulates the current rotation and output reading for various frequencies up to $50 \mathrm{kHz}$. Amplification was performed via a highperformance instrumentation amplifier (LMP8358).

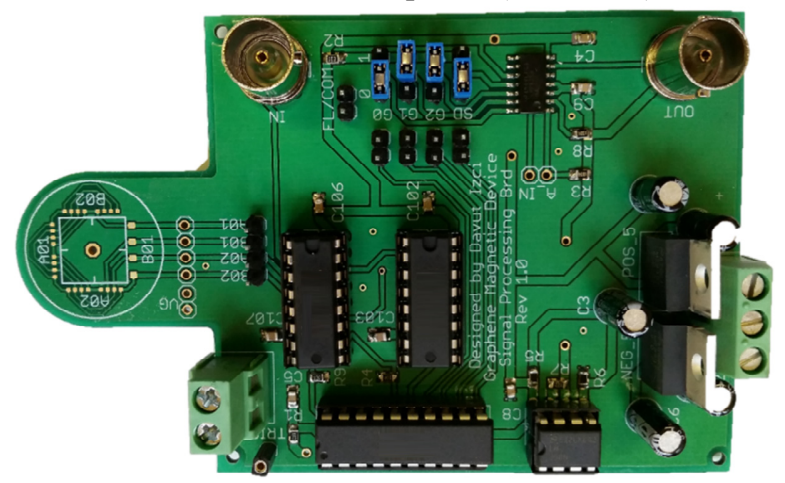

Fig. 6. Constructed system board PCB. The sensors can easily be mounted on the tongue shaped tip or can be remotely connected via specified pins provided. The on-board current biasing mechanism can be used for biasing the Hall elements. The PCB also allows a current source to be connected externally for biasing. 
The whole set-up was synchronized with a data acquisition card for obtaining the output and a LabVIEW interface was created for saving and analyzing the obtained data. The sensors can be integrated by being mounted on the board via the tongue shaped tip shown. Connections can also be made remotely using the relevant connection pins on the board. Assembling the driving and read-out system on a PCB is beneficial since there are challenges to integrate graphene on a single chip which encompasses all the electronic functionalities required for operation. As was demonstrated in a previous study [33], the complexity and temperature requirements of fabrication makes it difficult to integrate the graphene devices with a chip having biasing and read-out functionality.

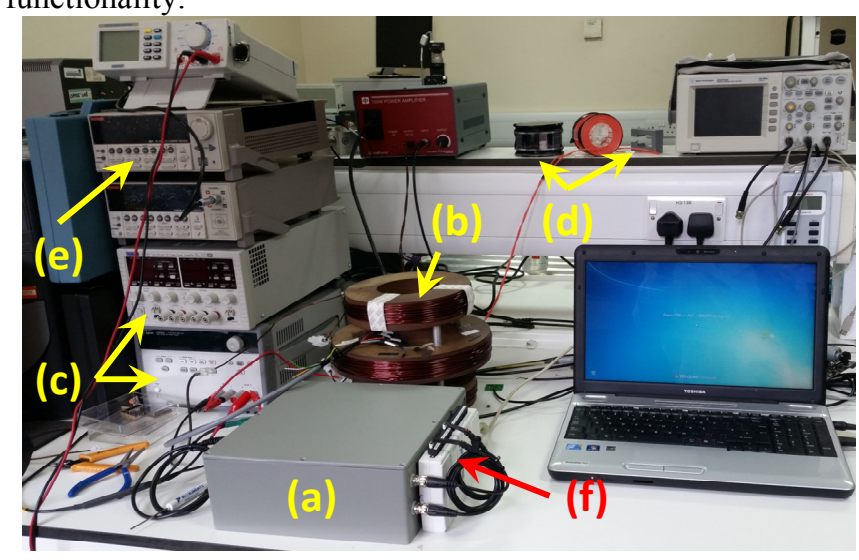

Fig. 7. Measurement setup including a Faraday box (a) for eliminating external noise sources, a Maxwell coil (b) with power supplies (c) and permanent rare earth magnets (d) for obtaining variable and constant magnetic fields uniformly and a Keithley 6221 current source (e) for biasing with DAQ device (f) for data acquisition.

\section{RESULTS}

Several micro-fabricated Hall elements with the same geometries, having length to width ratios of 3.5, were tested to observe the output characteristics of devices. Quantitative analysis was performed both under constant magnetic field with variable current and under constant current with variable magnetic field. The ability of detecting magnetic field change is one of the key parameters for determining the performance of Hall devices [5]. To achieve devices with the capability of low field sensing, the offset equivalent magnetic field needs to be optimized first. Reducing this parameter is important for sensitivity improvements and consistency. The offset equivalent magnetic field is defined as the ratio of offset voltage to the absolute sensitivity and given as:

$$
B_{o f f}=\frac{V_{o f f}}{S_{A}}
$$

where $B_{\text {off }}$ stands for offset equivalent magnetic field and $V_{\text {off }}$ represents offset voltage. $S_{A}$ represents the absolute sensitivity of a Hall device, one of the parameters used for sensitivity measurement, and it is given by the change in output voltage as a function of applied field under a certain biasing current. The latter term is given as:

$$
S_{A}=\left|\frac{V_{H}}{B_{y}}\right| .
$$

As shown in Fig. 8, a residual offset corresponding to an offset equivalent magnetic field value of $100 \mathrm{nT}$ was obtained [40]. Eliminating this considerably increases the sensitivity of the devices.

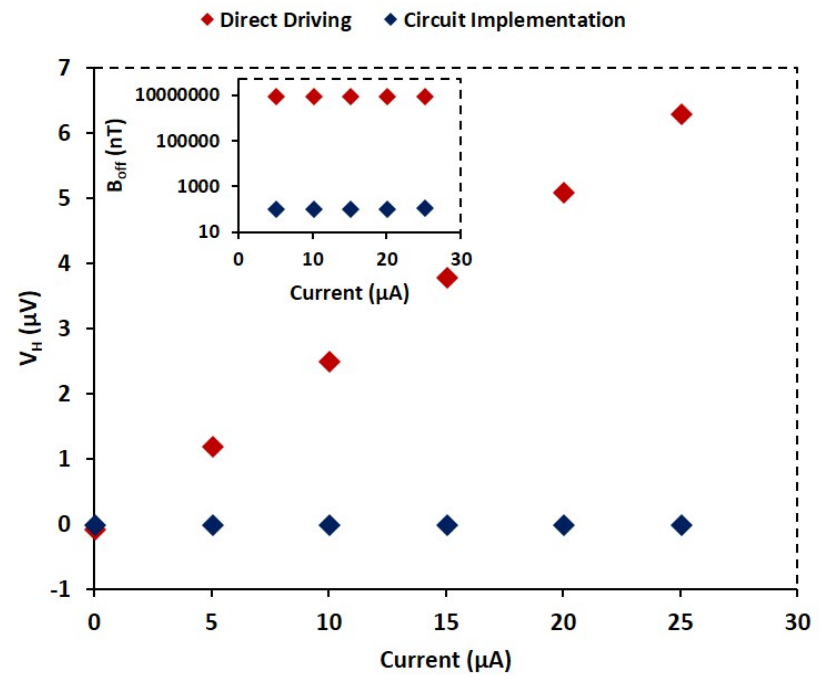

Fig. 8. Direct driving of the sensor under no magnetic field with varying current. The inset data shows residual magnetic offset values for nonprocessed and processed outputs, respectively.

Table 1 presents the comparison of residual offsets between the developed circuitry and some of the reported methods. The results show the promise of developed system for offset reduction.

TABLE 1. COMPARISON OF RESIDUAL OFFSETS

Offset Reduction Method

Residual Offset

\begin{tabular}{lc}
\hline Current Chopping (Ref [41]) & $31 \mu \mathrm{T}$ \\
\hline Correlated double sampling (Ref [42]) & $<200 \mu \mathrm{T}$ \\
\hline Orthogonal switching (Ref [43]) & $10 \mu \mathrm{T}$ \\
\hline This work & $100 \mathrm{nT}$ \\
\hline
\end{tabular}

Together with detection capability, a linear performance is desirable for practical implementation of Hall effect devices. As seen from Fig. 9 the Hall voltage of fabricated graphene devices shows good linearity with respect to both applied field strength and driving current. 

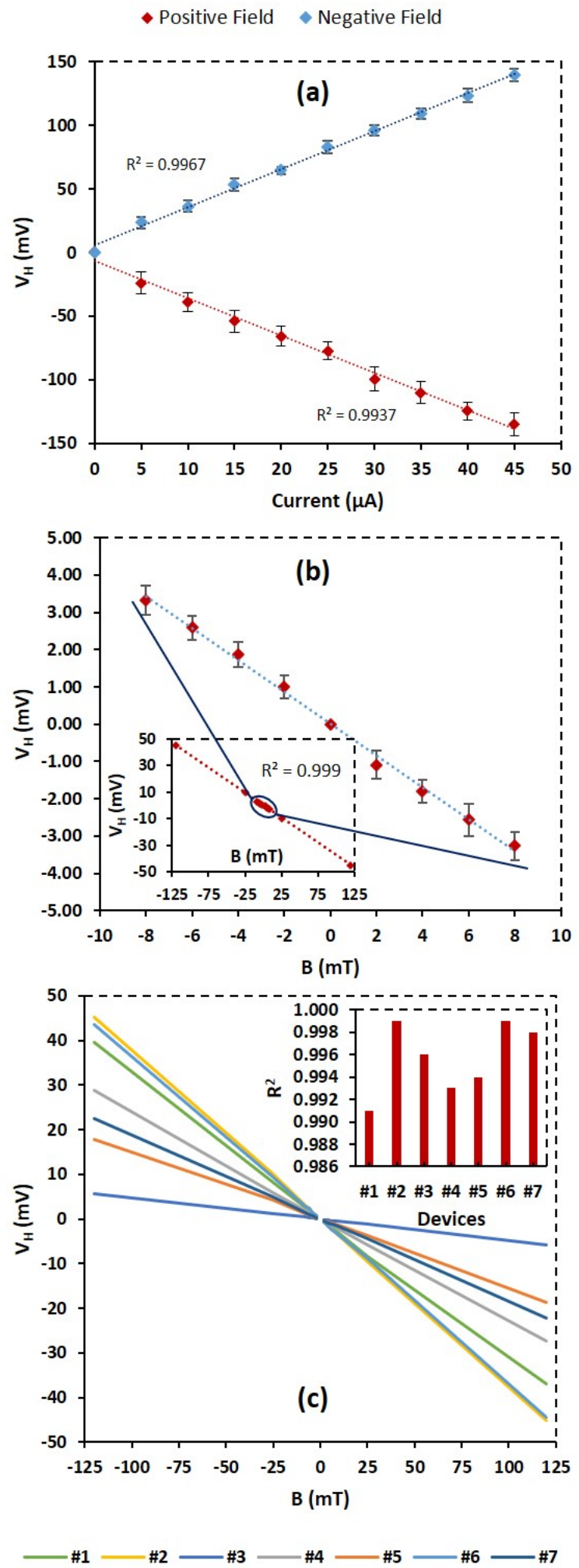

Fig. 9. Hall voltage under constant negative (red) and positive (blue) field strength of $120 \mathrm{mT}$ with variable driving current (a) and under variable magnetic field with constant driving current of $15 \mu \mathrm{A}(\mathrm{b})$. The inset of (b) shows a wider range. The repeatability $(n=3$ for (a) and $n=6$ for (b)) tests showed that devices are highly stable in terms of providing corresponding outputs. The response of the graphene sensors shows highly linear behavior (c). The inset of (c) shows corresponding linearity values across devices.
As seen from Fig. 9(c), the fabricated sensors showed a highly linear behavior. The demonstrated highly linear response $\left(\mathrm{R}^{2}>0.99\right)$ is comparable with those reported in previous studies on graphene devices [7, 33, 44].

Current-related sensitivity, $S_{I}$, is mostly used as a quantitative performance parameter for Hall devices. It is defined as the ratio of absolute sensitivity to applied current and given as:

$$
S_{I}=\left|\frac{S_{A}}{I_{X}}\right|
$$

where $I_{X}$ is the applied current value. For these devices, a maximum current related sensitivity of 2540 V/AT was obtained with no gate voltage applied. This current-related sensitivity is higher than the reported values shown in Table 2.

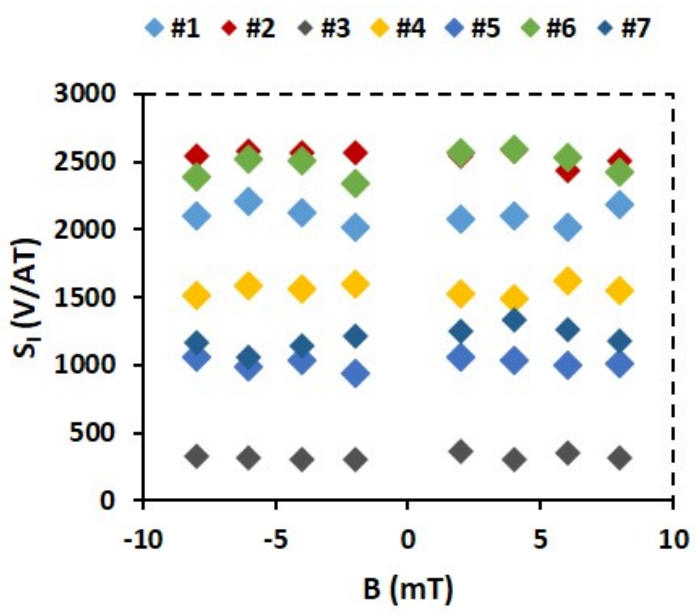

Fig. 10. Current-related sensitivities across different graphene Hall devices with the same geometry and sizes under the same operating conditions (15 $\mu \mathrm{A}$ biasing current and variable field).

As seen from Fig. 10, the sensitivity varies across devices. The sensitivity variations across those devices can be explained by non-uniform adhesion of graphene to the substrate, defects and ruptures or residuals caused the by fabrication process [45]. Differences in the input resistances of devices may also play a role in sensitivity variations [46]. The sensitivity variation may seem to be contradictory to what Raman spectroscopy shows (Fig. 3) since a high-quality graphene film with low defect is presented. However, the sensitivity of devices would vary depending where the defect is located. For example, a low defect on the contacts region may not cause a considerable reduction in the sensitivity whereas a low defect on an active area would reduce the sensitivity incredibly. Nevertheless, thanks to the excellent electrical properties of graphene, fabricated devices with even the worst performance (323 V/AT) still provide better sensitivities compared to $\mathrm{Si}$ based competitors (310 V/AT) [34]. It is worth to note that the sensor-to-sensor variation could be calibrated by applying a gate voltage during the measurements. Because applying a gate voltage would cause a change in the density of charge carriers [47] which would consequently lead to a change in the sensitivity. This has already been demonstrated in a previous work [7] by increasing the sensitivity from 550 V/AT (applying no gate 
voltage) to about $1500 \mathrm{~V} / \mathrm{AT}$ (applying a gate voltage of 10.5 V).

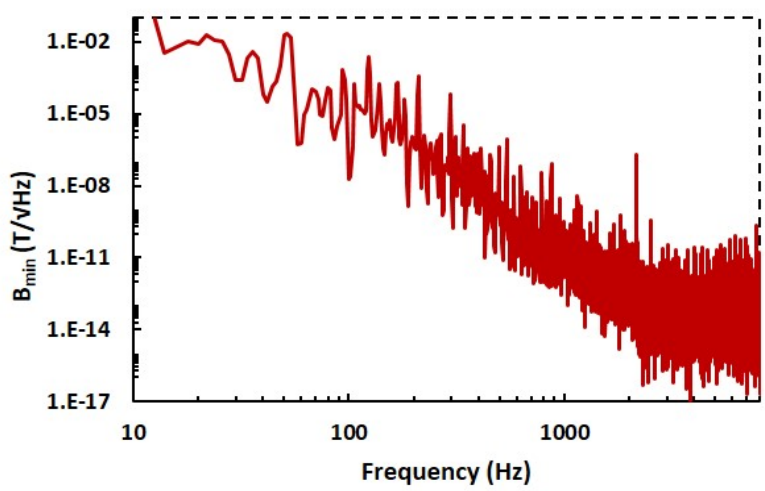

Fig. 11. Magnetic field resolution of a graphene Hall sensor as a function of frequency.

In addition of representing high sensitivity, graphene also has low noise intrinsically [48]. Having a combination of low noise and high sensitivity leads to devices with high resolutions. Magnetic field resolution is the parameter that is used for determining the minimum detectable field capability of the sensor and given as;

$$
B_{\text {min }}=\frac{\sqrt{4 k_{B} T R_{s} \Delta f}}{R_{H} I_{x}}
$$

where, $k_{B}$ is the Boltzmann constant, $T$ temperature, $R_{s}$ series resistance, $R_{H}$ Hall coefficient and $\Delta f$ measurement bandwidth. The minimum detectable magnetic field parameter can also be determined according to (5), where $B_{\min }$ is the field resolution and $V_{n}$ represents the spectral density of the voltage noise.

$$
B_{\text {min }}=\left|\frac{V_{n}}{S_{A}}\right| \text {. }
$$

The latter equation states that the resolution can be calculated by dividing the noise spectra by absolute sensitivity. To determine this value, the FFT tool of LabVIEW software was used to measure voltage noise spectra by adopting a National Instruments' data acquisition device (NI-DAQ USB-6211). The data of measured noise spectra $(\mathrm{V} / \mathrm{V} \mathrm{Hz})$ was divided by absolute sensitivity, during the operation, to obtain the minimum detectable field $(\mathrm{T} / \sqrt{\mathrm{Hz}})$. Magnetic field resolution of the sensor with respect to frequency was obtained as shown in Fig. 11. It is worth to note that the thermal noise is usually the dominant noise at high frequencies, therefore, the minimum detectable field can be defined by thermal noise region within a noise spectrum. That means the maximum achievable value within the thermal noise region is considered as the magnetic resolution of a device. As seen in Fig. 11, the noise level decreases with respect to increasing frequency, representing a $1 / \mathrm{f}$ dependence. Around $2 \mathrm{kHz}$, the noise level is dominated by thermal noise only. A minimum field resolution of $162 \mathrm{nT} / \sqrt{\mathrm{Hz}}$ was extracted from fast Fourier transform measurements and this compares favorably to previous reported values given in Table 2.s
TABLE 2. COMPARISON OF CURRENT RELATED SENSITIVITIES AND MINIMUM DETECTABLE FIELD RESOLUTIONS

\begin{tabular}{lccc}
\hline \multicolumn{1}{c}{ Material } & $\begin{array}{c}\mathrm{S}_{\mathrm{I}} \\
\text { (V/AT) }\end{array}$ & $\begin{array}{c}\mathrm{B}_{\min } \\
(\mathrm{nT} / \sqrt{\mathrm{Hz}})\end{array}$ & $\begin{array}{c}\text { Linearity } \\
\text { Error (\%) }\end{array}$ \\
\hline CVD graphene (This work) & 2540 & 162 & $1 \%$ \\
\hline CVD graphene (Ref [26]) & 2093 & 100 & $4 \%$ \\
\hline CVD graphene (Ref [49]) & 1800 & 20000 & - \\
\hline CVD graphene (Ref [7]) & 1200 & 800 & $2 \%$ \\
\hline CVD graphene (Ref [45]) & 1200 & 43000 & - \\
\hline CVD graphene (Ref [27]) & 800 & 500 & - \\
\hline Epitaxial graphene (Ref [50]) & 1021 & 2500 & - \\
\hline 2DEG (Ref [51]) & 357 & 500 & $3 \%$ \\
\hline Silicon (Ref [52]) & 175 & 200 & - \\
\hline Silicon (Ref [53]) & 143 & 250 & - \\
\hline
\end{tabular}

\section{CONCLUSION}

Graphene based micro-Hall elements were fabricated using microfabrication techniques in parallel to realization of an electronic system for driving and processing the signal, resulting in a highly sensitive magnetometer system. The sensor output was corrected with a high correction ratio and the graphene devices demonstrated excellent behavior in terms of linearity, sensitivity and resolution. The performance values, in terms of current related sensitivity and magnetic field resolution, of $2540 \mathrm{~V} / \mathrm{AT}$ and $162 \mathrm{nT} / \sqrt{\mathrm{Hz}}$ were obtained respectively, outperforming the competitor Hall effect devices fabricated from graphene. The results show that graphene, with suitable processing electronics, is an excellent candidate for magneto-sensing applications of low field strengths.

\section{ACKNOWLEDGMENT}

Davut Izci acknowledges The Council of Higher Education in Turkey for the scholarship award.

\section{REFERENCES}

[1] J. Lenz, and A. S. Edelstein, "Magnetic sensors and their applications," IEEE Sensors Journal, vol. 6, no. 3, pp. 631-649, Jun, 2006.

[2] L. Jogschies, D. Klaas, R. Kruppe et al., "Recent Developments of Magnetoresistive Sensors for Industrial Applications," Sensors (Basel), vol. 15, no. 11, pp. 28665-89, Nov 12, 2015.

[3] M. I. Faley, E. A. Kostyurina, K. V. Kalashnikov et al., "Superconducting Quantum Interferometers for Nondestructive Evaluation," Sensors (Basel), vol. 17, no. 12, pp. 2798, Dec 6, 2017.

[4] A. Jitariu, C. Ghemes, N. Lupu et al., "Magnetic particles detection by using spin valve sensors and magnetic traps," Aip Advances, vol. 7, no. 5, pp. 056616, May, 2017.

[5] R. S. Popović, Hall effect devices, 2nd ed.: Philadelphia : Institute of Physics Pub., 2004.

[6] J. E. Lenz, "A Review of Magnetic Sensors," Proceedings of the IEEE, vol. 78, no. 6, pp. 973-989, Jun, 1990.

[7] H. Xu, Z. Zhang, R. Shi et al., "Batch-fabricated high-performance graphene Hall elements," Sci Rep, vol. 3, pp. 1207, 2013.

[8] E. Ramsden, Hall-Effect Sensors - Theory and Application, 2nd ed.: Elsevier, 2006.

[9] A. Sandhu, Y. Kumagai, A. Lapicki et al., "High efficiency Hall effect micro-biosensor platform for detection of magnetically 
labeled biomolecules," Biosens Bioelectron, vol. 22, no. 9-10, pp. 2115-20, Apr 15, 2007

[10] K. Togawa, H. Sanbonsugi, A. Sandhu et al., "Detection of magnetically labeled DNA using pseudomorphic $\mathrm{AlGaAs} / \mathrm{InGaAs} / \mathrm{GaAs}$ heterostructure micro-Hall biosensors," Journal of Applied Physics, vol. 99, no. 8, Apr 15, 2006.

[11] G. Mihajlovic, P. Xiong, S. von Molnar et al., "Detection of single magnetic bead for biological applications using an InAs quantumwell micro-Hall sensor," Applied Physics Letters, vol. 87, no. 11, Sep 12, 2005.

[12] D. Issadore, J. Chung, H. Shao et al., "Ultrasensitive clinical enumeration of rare cells ex vivo using a micro-hall detector," $\mathrm{Sci}$ Transl Med, vol. 4, no. 141, pp. 141ra92, Jul 4, 2012.

[13] B. T. Dalslet, C. D. Damsgaard, M. Donolato et al., "Bead magnetorelaxometry with an on-chip magnetoresistive sensor,' Lab Chip, vol. 11, no. 2, pp. 296-302, Jan 21, 2011.

[14] F. W. Østerberg, G. Rizzi, T. Zardan Gomez de la Torre et al., "Measurements of Brownian relaxation of magnetic nanobeads using planar Hall effect bridge sensors," Biosens Bioelectron, vol 40, no. 1, pp. 147-52, Feb 15, 2013.

[15] P. Murali, A. M. Niknejad, and B. E. Boser, "CMOS Microflow Cytometer for Magnetic Label Detection and Classification," Ieee Journal of Solid-State Circuits, vol. 52, no. 2, pp. 543-555, Feb, 2017.

[16] D. P. Renella, S. Spasic, S. Dimitrijevic et al., "An overview of commercially available teslameters for applications in modern science and industry," Acta IMEKO, vol. 6, no. 1, pp. 43-49, 2017.

[17] M.-A. Paun, J.-M. Sallese, and M. Kayal, "Hall Effect Sensors Design, Integration and Behavior Analysis," Journal of Sensor and Actuator Networks, vol. 2, no. 1, pp. 85-97, 2013.

[18] N. A. A. Ghany, S. A. Elsherif, and H. T. Handal, "Revolution of Graphene for different applications: State-of-the-art," Surfaces and Interfaces, vol. 9, pp. 93-106, Dec, 2017.

[19] R. Kumar, R. Singh, D. Hui et al., "Graphene as biomedical sensing element: State of art review and potential engineering applications," Composites Part B-Engineering, vol. 134, pp. 193206, Feb 1, 2018.

[20] I. W. Frank, D. M. Tanenbaum, A. M. Van der Zande et al. "Mechanical properties of suspended graphene sheets," Journal of Vacuum Science \& Technology B, vol. 25, no. 6, pp. 2558-2561, Nov, 2007.

[21] N. O. Weiss, H. Zhou, L. Liao et al., "Graphene: an emerging electronic material," Adv Mater, vol. 24, no. 43, pp. 5782-825, Nov $14,2012$.

[22] C. Lee, X. Wei, J. W. Kysar et al., "Measurement of the elastic properties and intrinsic strength of monolayer graphene," Science, vol. 321 , no. 5887 , pp. 385-8, Jul 18, 2008.

[23] A. K. Geim, "Graphene: status and prospects," Science, vol. 324, no. 5934, pp. 1530-4, Jun 19, 2009.

[24] K. I. Bolotin, K. J. Sikes, Z. Jiang et al., "Ultrahigh electron mobility in suspended graphene," Solid State Communications, vol. 146, no. 9-10, pp. 351-355, Jun, 2008.

[25] B. Y. Chen, L. Huang, X. M. Ma et al., "Exploration of sensitivity limit for graphene magnetic sensors," Carbon, vol. 94, pp. 585589, Nov, 2015.

[26] L. Huang, Z. Y. Zhang, B. Y. Chen et al., "Ultra-sensitive graphene Hall elements," Applied Physics Letters, vol. 104, no. 18, May 5, 2014.

[27] H. L. Xu, L. Huang, Z. Y. Zhang et al., "Flicker noise and magnetic resolution of graphene hall sensors at low frequency," Applied Physics Letters, vol. 103, no. 11, Sep 9, 2013.

[28] Y. H. Zheng, L. Huang, Z. Y. Zhang et al., "Sensitivity enhancement of graphene Hall sensors modified by singlemolecule magnets at room temperature," Rsc Advances, vol. 7, no. 4, pp. 1776-1781, 2017.

[29] E. Kojima, K. Kano, H. Wado et al., "Magnetic Field Sensor of Graphene for Automotive Applications," in SAE Technical Paper Series, 2017.

[30] Z. Wang, M. Shaygan, M. Otto et al., "Flexible Hall sensors based on graphene," Nanoscale, vol. 8, no. 14, pp. 7683-7, Apr 14, 2016. R. K. Rajkumar, A. Asenjo, V. Panchal et al., "Magnetic scanning gate microscopy of graphene Hall devices (invited)," Journal of Applied Physics, vol. 115, no. 17, May 7, 2014.
[32] S. Pisana, P. M. Braganca, E. E. Marinero et al., "Graphene Magnetic Field Sensors," IEEE Transactions on Magnetics, vol. 46, no. 6, pp. 1910-1913, Jun, 2010.

[33] L. Huang, H. Xu, Z. Zhang et al., "Graphene/Si CMOS hybrid hall integrated circuits,” Sci Rep, vol. 4, pp. 5548, Jul 7, 2014.

[34] Y. Xu, H. B. Pan, S. Z. He et al., "A highly sensitive CMOS digital Hall sensor for low magnetic field applications," Sensors (Basel), vol. 12, no. 2, pp. 2162-74, 2012.

[35] A. C. Ferrari, and D. M. Basko, "Raman spectroscopy as a versatile tool for studying the properties of graphene," Nat Nanotechnol, vol. 8, no. 4, pp. 235-46, Apr, 2013.

[36] C. C. Enz, and G. C. Temes, "Circuit techniques for reducing the effects of op-amp imperfections: Autozeroing, correlated double sampling, and chopper stabilization," Proceedings of the IEEE, vol. 84, no. 11, pp. 1584-1614, Nov, 1996.

[37] X. Zhou, C. C. Huang, and D. A. Hall, "Giant Magnetoresistive Biosensor Array for Detecting Magnetorelaxation," IEEE Trans Biomed Circuits Syst, vol. 11, no. 4, pp. 755-764, Aug, 2017.

[38] S. Gambini, K. Skucha, P. P. Liu et al., "A 10 kPixel CMOS Hall Sensor Array With Baseline Suppression and Parallel Readout for Immunoassays," IEEE Journal of Solid-State Circuits, vol. 48, no. 1, pp. 302-317, Jan, 2013.

[39] R. Steiner, C. Maier, A. Haberli et al., "Offset reduction in Hall devices by continuous spinning current method," Sensors and Actuators a-Physical, vol. 66, no. 1-3, pp. 167-172, Apr 1, 1998.

[40] D. Izci, C. Dale, N. Keegan et al., "Design and construction of a high sensitive graphene magnetosensing system," 2017 IEEE Sensors Conerence, pp. 1-3, 2017.

[41] M. Li, and D. A. Horsley, "Offset Suppression in a Micromachined Lorentz Force Magnetic Sensor by Current Chopping," Journal of Microelectromechanical Systems, vol. 23, no. 6, pp. 1477-1484, Dec, 2014.

[42] C. Xiaoqing, X. Yue, X. Xiaopeng et al., "A novel Hall dynamic offset cancellation circuit based on four-phase spinning current technique," Semiconductor Technology International Conference (CSTIC), 2015 China, pp. 1-3, 2015.

[43] Z. Stoessel, and M. Resch, "Flicker noise and offset suppression in symmetric hall plates," Sensors and Actuators A: Physical, vol. 3738, pp. 449-452, 1993/06/01/, 1993.

[44] L. Huang, Z. Zhang, Z. Li et al., "Multifunctional graphene sensors for magnetic and hydrogen detection," ACS Appl Mater Interfaces, vol. 7, no. 18, pp. 9581-8, May 13, 2015.

[45] C. C. Tang, M. Y. Li, L. J. Li et al., "Characteristics of a sensitive micro-Hall probe fabricated on chemical vapor deposited graphene over the temperature range from liquid-helium to room temperature," Applied Physics Letters, vol. 99, no. 11, Sep 12, 2011.

[46] N. Haned, and M. Missous, "Nano-tesla magnetic field magnetometry using an InGaAs-AlGaAs-GaAs 2DEG Hall sensor," Sensors and Actuators a-Physical, vol. 102, no. 3, pp. 216-222, Jan 1, 2003

[47] F. Schwierz, "Graphene transistors," Nat Nanotechnol, vol. 5, no. 7, pp. 487-96, Jul, 2010.

[48] J. S. Moon, D. Curtis, D. Zehnder et al., "Low-Phase-Noise Graphene FETs in Ambipolar RF Applications," IEEE Electron Device Letters, vol. 32, no. 3, pp. 270-272, Mar, 2011.

[49] S. Sonusen, O. Karci, M. Dede et al., "Single layer graphene Hall sensors for scanning Hall probe microscopy (SHPM) in 3-300 K temperature range," Applied Surface Science, vol. 308, pp. 414418, Jul 30, 2014

[50] V. Panchal, K. Cedergren, R. Yakimova et al., "Small epitaxial graphene devices for magnetosensing applications," Journal of Applied Physics, vol. 111, no. 7, Apr 1, 2012.

[51] O. Kazakova, J. C. Gallop, D. C. Cox et al., "Optimization of 2DEG InAs/GaSb Hall Sensors for Single Particle Detection," IEEE Transactions on Magnetics, vol. 44, no. 11, pp. 4480-4483, Nov, 2008.

[52] P. A. Besse, G. Boero, M. Demierre et al., "Detection of a single magnetic microbead using a miniaturized silicon Hall sensor," Applied Physics Letters, vol. 80, no. 22, pp. 4199-4201, Jun 3, 2002.

[53] D. S. Mellet, and M. du Plessis, "A novel CMOS Hall effect sensor," Sensors and Actuators a-Physical, vol. 211, pp. 60-66, May 1, 2014. 


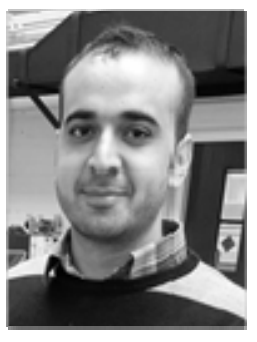

Davut Izci received his B.Sc. in Electrical \& Electronic Engineering in 2009 from Dicle University, Turkey and his M.Sc. in Mechatronics from Newcastle University, UK in 2013.

He worked for leading tech companies as an engineer and took management roles for more than two years after his graduation. $\mathrm{He}$ is currently a Ph.D. candidate in Newcastle University, UK, and an academic at Batman University, Turkey, working on development of graphene-based sensing applications.

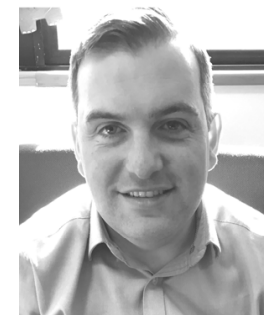

Carl Dale received a B.Sc in Applied Biochemistry in 2005 from Liverpool John Moores University, U.K.; an M.Res. in Medical and Molecular Biosciences in 2007 from Newcastle University, U.K.; followed by a Ph.D. degree in Microsystems Engineering in 2012 from Newcastle University.

He is currently a Postdoctoral Research Associate within the Institute of Cellular Medicine at Newcastle University. His research interests focus on the development and exploitation of novel technologies for biological sensing applications.

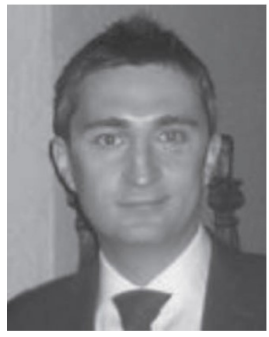

Neil Keegan received a B.Sc in Biochemistry in 1997 from Newcastle University, UK. He subsequently gained four years industrial experience with Pfizer pharmaceuticals, UK, before returning to Newcastle University to obtain a Medical Research Council sponsored Ph.D. degree in Biomedical Nanotechnology in 2005. After a three year Research Associate position and a further three year position as a Senior Research Associate, working on biological sensor systems, he obtained an academic position within the Institute of Cellular Medicine at Newcastle University. He is currently a Senior Lecturer with research interests in general diagnostics, biosensors and biological sensor systems.

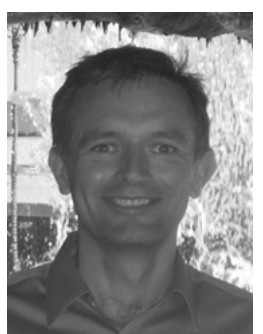

John Hedley received a B.Sc. in Astronomy and Astrophysics in 1991 from Newcastle University, U.K., followed by a Ph.D. degree in Atomic Physics from Newcastle University in 1996.

After three Postdoctoral Researcher positions in MEMS design, fabrication, and testing, he obtained an academic position within the School of Engineering at Newcastle University where he is now Senior Lecturer. His research interests are in microsystems development and sensing applications utilizing graphene. 